A note on the Shapley value for characteristic functions on bipartitions

Sander Muns 



\title{
A note on the Shapley value for characteristic functions on bipartitions
}

\author{
Sander Muns*
}

August 28, 2011

\begin{abstract}
We consider a cooperative game with a bipartition that indicates which players are participating. This paper provides an analytical solution for the Shapley value when the worth of a coalition only depends on the number of participating coalition players. The computational complexity grows linearly in the number of players, which contrasts with the usual exponential increase. Our result remains true when we introduce (i) randomization of the bipartition, and (ii) randomly draw a characteristic function.
\end{abstract}

Keywords: Shapley value, computational complexity, bipartition

\section{JEL classification: C71}

\section{Introduction}

A cooperative game $(N, v)$ consists of a player set $N$ and a characteristic function $v \in \mathscr{F}^{N}$, where $\mathscr{F}^{N}$ denotes the class of functions $v: 2^{N} \rightarrow \mathbb{R}$ that satisfy $v(\emptyset)=0$. The worth $v(N)$ of the game is the worth generated by the coalition of all $n$ players in $N$. The Shapley value $\phi: \mathscr{F}^{N} \rightarrow \mathbb{R}^{n}$ (Shapley [1953]) is an allocation of this worth. For any player $i \in N$ :

$$
\phi_{i}(v)=\sum_{\{S: i \notin S\}} \frac{|S| !(n-|S|-1) !}{n !}[v(S \cup i)-v(S)]
$$

where $|S|$ is the number of players in $S$. It follows from $(1)$ that the Shapley value is a linear function:

$$
\phi(v)=\alpha \phi(w)+\beta \phi(z) \quad \alpha, \beta \in \mathbb{R} \quad w, z \in \mathscr{F}^{N}
$$

where $v \in \mathscr{F}^{N}$ is for each $S \subseteq N$ defined as $v(S)=\alpha w(S)+\beta z(S)$. The Shapley value is fair in the sense that it satisfies several axiomatizations (see Winter [2002] for an overview). For instance, Myerson [1980] shows that the Shapley value is the unique allocation that satisfies the following two axioms:

\footnotetext{
*Erasmus University Rotterdam, Tinbergen Institute, and CPB Netherlands Bureau for Economic Policy Analysis, e-mail: muns @ ese.eur.nl I am grateful to Gerard van der Laan and Casper de Vries for helpful comments.
} 


\section{(i) Efficiency}

The sum of the allocated values adds up to the total worth of the game:

$$
\sum_{i \in N} \phi_{i}(v)=v(N)
$$

\section{(ii) Balanced contributions}

For any game $(N, v)$ with players $i$ and $j(i \neq j)$, the loss that player $i$ incurs after player $j$ is removed from the game is equal to the loss that player $j$ incurs after player $i$ is removed from the game:

$$
\phi_{i}(v)-\phi_{i}\left(\left.v\right|_{N \backslash j}\right)=\phi_{j}(v)-\phi_{j}\left(\left.v\right|_{N \backslash i}\right)
$$

where $\left.v\right|_{S}$ is the restriction of $v$ to the subsets of $S$, and $\phi_{i}\left(\left.v\right|_{S}\right)$ denotes the allocation to player $i$ in the subgame $\left(S,\left.v\right|_{S}\right)$.

For each player, the number of possible subsets $S$ in 11 equals $2^{n-1}$. Thus the computational complexity increases exponentially in the number of players (see e.g. Faigle and Kern [1992]). Several papers have proposed approximation techniques where the computational complexity increases only linearly in the number of players (e.g., Fatima et al [2008] and Castro et al [2009]). Our focus is on an exact determination of the Shapley value for a specific class of characteristic functions.

Megiddo [1978] shows that the Shapley value is obtained in $O(n)$ computations for games where players are nodes in a tree. Granot et al [2002] extend this result to heterogeneous preferences of the players. A computational complexity of $O\left(n^{2}\right)$ is found in Deng and Papadimitriou [1994] for a game in which the players are nodes in an undirected graph. This computational complexity can never be smaller for this class of games since the number of arcs is $O\left(n^{2}\right)$ for a complete graph.

For some classes of characteristic functions, the computational complexity of the Shapley value is polynomial in the number of players. For example, the Shapley value of a weighted majority game is computed in $O\left(n^{3}\right)$ computations in Algaba et al [2003]. Another example is in Littlechild and Owen [1973], who show that the computational complexity is $O(n)$ if the worth of a coalition equals the maximal worth of a single coalition player.

We identify in Section 2 another class which has the tractable linear computational complexity. This class consists of characteristic functions where the worth only depends on the number of participating coalition players. In Section 3 it is shown that the linearity of the Shapley value implies that the computational complexity is unaffected when we (i) randomize participations, and (ii) randomly draw a characteristic function. Section 4 illustrates the results by means of an example.

\section{Deterministic setting}

We start with a deterministic version of our main result that the computational complexity is linear in the number of players when the characteristic function depends on the number of participating players. Consider a game $(N, v)$ with $n$ players in $N$. A bipartition on $N$ indicates which players are participating. For notational convenience, we 
use the participation vector $B \in[0,1]^{n}$ to characterize the bipartition:

$$
B_{i}= \begin{cases}0 & \text { if } i \text { is a null player } \\ 1 & \text { if } i \text { is a participating player }\end{cases}
$$

The total number of participating players in the coalition $S$ is given by $\kappa_{S}=\sum_{i \in S} B_{i}$, and we define $\kappa:=\kappa_{N}$ as the total number of participating players. The following proposition enables us to obtain the Shapley value in $O(n)$ computations for a special class of characteristic functions.

Proposition 1. Consider the setting described above. If the characteristic function $v$ is of the type

$$
v(S)=\kappa_{S} f\left(\kappa_{S}\right) \quad S \in 2^{N}
$$

where $f:\{0,1, \ldots, n\} \rightarrow \mathbb{R}$, then the Shapley value of player $i$ equals

$$
\phi_{i}(v)=B_{i} f(\kappa)
$$

This means that the Shapley value of player i equals the fraction $\frac{B_{i}}{\kappa}$ of the worth $v(N)$ of the game.

Proof. We show that the allocation $\phi(v)$ satisfies the efficiency axiom as well as the balanced contributions axiom. It is easy to see that $\phi(v)$ satisfies the efficiency axiom because

$$
\sum_{i \in N} \phi_{i}(v)=\sum_{i \in N} B_{i} f(\kappa)=\kappa f(\kappa)=v(N)
$$

For any $i, j \in N(i \neq j)$, we have $\phi_{i}(v)=B_{i} f(\kappa)$ and $\phi_{i}\left(\left.v\right|_{N \backslash j}\right)=B_{i} f\left(\kappa-B_{j}\right)$, such that the balanced contributions axiom is necessarily satisfied if

$$
B_{i} f(\kappa)-B_{i} f\left(\kappa-B_{j}\right)=B_{j} f(\kappa)-B_{j} f\left(\kappa-B_{i}\right)
$$

Obviously, this equation holds for any of the four possibilities of the pair $\left(B_{i}, B_{j}\right)$. This implies that $\phi(v)$ satisfies the balanced contributions axiom for any game $(N, v)$, as required.

Proposition 1 makes clear that the vector $\phi(v)$ is obtained by first computing $f(\kappa)$ and subsequently $B_{i} f(\kappa)$ for each player $i \in N$. This results in a computational complexity that grows linearly in the number of players in $N$.

The class of characteristic functions in Proposition 1 nests the class of voting games where each player has the same weight. However, it cannot be generalized to weighted Shapley values. For an arbitrary nonzero weight vector $w \in \mathbb{R}^{n}(w \neq 0)$, the balanced contributions axiom changes then into (see Kalai and Samet [1987]):

$$
w_{j}\left[\phi_{i}(v)-\phi_{i}\left(\left.v\right|_{N \backslash j}\right)\right]=w_{i}\left[\phi_{j}(v)-\phi_{j}\left(\left.v\right|_{N \backslash i}\right)\right]
$$

The case $w_{i} \neq 0$ for exactly one player $i$ is neglected, because the weight vector would become the zero vector in the game $\left(N \backslash i,\left.v\right|_{N \backslash i}\right)$. We write the allocation in the functional form $\phi_{i}\left(\left.v\right|_{S}\right)=w_{i} \gamma_{i}^{S} B_{i} f\left(\kappa_{S}\right)$ and show that $\gamma_{i}^{S}$ depends on the functional form of $f$. This makes the simple closed form solution in Proposition 1 infeasible. The efficiency axiom imposes

$$
\sum_{i \in N} w_{i} \gamma_{i}^{N} B_{i} f(\kappa)=\kappa f(\kappa)
$$


If $\gamma_{i}^{N}$ does not depend on the functional form of $f$, then we need to have (i) $\gamma_{i}^{N}=\frac{1}{w_{i}}$ or $(i i) \gamma_{i}^{N}=\kappa\left(\sum_{k \in N} w_{k} B_{k}\right)^{-1}$. By the imposed functional form, the balanced contributions axiom 22 is satisfied for $\left(B_{i}, B_{j}\right)=(1,1)$ when

$$
w_{j} w_{i}\left[\gamma_{i}^{N} f(\kappa)-\gamma_{i}^{N \backslash j} f(\kappa-1)\right]=w_{i} w_{j}\left[\gamma_{j}^{N} f(\kappa)-\gamma_{j}^{N \backslash i} f(\kappa-1)\right]
$$

The latter equation is only satisfied for any function $f$ if $\gamma_{i}^{N}=\gamma_{j}^{N}$ and $\gamma_{i}^{N \backslash j}=\gamma_{j}^{N \backslash i}$. This shows that (i) and (ii) are both inappropriate, except for the special case $w_{1}=\ldots=w_{n} \neq 0$ which corresponds with Proposition 1 .

\section{Stochastic setting}

We allow the participation vector $B$ to be a random vector. Besides the fact that the outcome $B_{i}$ of player $i$ becomes stochastic, the outcome $B_{i}$ can be correlated with the outcome $B_{j}$ of another player $j$. We assume that $T$ different realizations of the elements in $B$ represent the probability distribution of $B$.

First, the characteristic function is some given deterministic function. Then, we generalize the setting to a randomization over a set of characteristic functions.

Deterministic characteristic function The following proposition extends Proposition 1 to stochastic participation vectors.

Proposition 2. Suppose that the characteristic function $v$ of the game $(N, v)$ is defined as

$$
v(S)=E\left[\kappa_{S} f\left(\kappa_{S}\right)\right]
$$

where $f:\{0,1, \ldots, n\} \rightarrow \mathbb{R}$, and the expectation is with respect to the realization of the random vector $B$. The Shapley value of player $i$ is then

$$
\phi_{i}(v)=E\left[B_{i} f(\kappa)\right]
$$

In words, the Shapley value of player $i$ equals the expectation of the fraction $\frac{B_{i}}{\kappa}$ of the realization of the worth function $\kappa f(\kappa)$.

Proof. Along the lines of the proof of Proposition 1, we show that the allocation of $\phi(v)$ satisfies the efficiency axiom as well as the balanced contributions axiom. It is easy to see that $\phi(v)$ satisfies the efficiency axiom because by the linearity of the expectations operator

$$
\sum_{i \in N} \phi_{i}(v)=E\left[\sum_{i \in N} B_{i} f(\kappa)\right]=E[\kappa f(\kappa)]=v(N)
$$

For any $i, j \in N(i \neq j)$, we have $\phi_{i}(v)=E\left[B_{i} f(\kappa)\right]$ and $\phi_{i}\left(\left.v\right|_{N \backslash j}\right)=E\left[B_{i} f\left(\kappa-B_{j}\right)\right]$. Then, the balanced contributions axiom is necessarily satisfied if for any realization $\hat{B}$ of $B$ :

$$
\hat{B}_{i} f(\hat{\kappa})-\hat{B}_{i} f\left(\hat{\kappa}-\hat{B}_{j}\right)=\hat{B}_{j} f(\hat{\kappa})-\hat{B}_{j} f\left(\hat{\kappa}-\hat{B}_{i}\right)
$$

where $\hat{\kappa}=\sum_{i \in N} \hat{B}_{i}$. Obviously, this equation holds for any of the four possible realizations of the pair $\left(\hat{B}_{i}, \hat{B}_{j}\right)$, and so for any realization $\hat{B}$ of $B$. Therefore, this equation is still valid if we take the expectation with respect to $B$. This implies that $\phi(v)$ satisfies the balanced contributions axiom, as required. 
Since we compute $B_{i} f(\kappa)$ for each of the $n$ players and each of the $T$ realizations of $B$, it is straightforward that the computational complexity of $\phi(v)$ grows linearly in $n$ as well as in $T$. This result can be generalized to the setting where the characteristic function $v$ admits $v(S)=\sum_{t=1}^{T} \kappa_{S}^{(t)} f\left(\kappa_{S}^{(t)}\right) h\left(t, B^{(t)}\right)$ for some $h:\{1, \ldots, T\} \times$ $[0,1]^{n} \rightarrow \mathbb{R}$, where $B^{(t)}$ is the $t$-th realization of $B$ and $\kappa^{(t)}=\sum_{i \in N} B_{i}^{(t)}$. The Shapley value of player $i$ is then given by $\phi_{i}(v)=\sum_{t=1}^{T} B_{i}^{(t)} f\left(\kappa^{(t)}\right) h\left(t, B^{(t)}\right){ }^{1}$ Proposition 2 refers to the special case $h \equiv \frac{1}{T}$. Notice that the characteristic function and the Shapley value remain deterministic after introducing randomization in $B$.

Randomization over characteristic functions We extend the stochastic setting to a randomization over a set of characteristic functions. The set $\mathscr{V}$ contains all possible realizations $\hat{v}: 2^{N} \rightarrow \mathbb{R}$ of the characteristic function $v$. The probability measure $P$ is defined on the elements of $\mathscr{V}$. The characteristic function $\bar{v}: 2^{N} \rightarrow \mathbb{R}$ of the composed game $(N, \bar{v})$ is defined as $\bar{v}(S)=E_{v}[v(S)]$, where $E_{v}$ denotes the expectation with respect to the realization of $v$. In other words, the characteristic function $\bar{v}$ attaches the weight $P(v=\hat{v})$ to the outcome $\hat{v}$ of $v$.

The player set $N$ is the same for each $\hat{v} \in \mathscr{V}$. In this way, the dimension of the allocation vector $\phi(\hat{v})$ that determines the Shapley value is the same for each $\hat{v} \in \mathscr{V}$. The distribution of $B$ is allowed to depend on $\hat{v}$. A sample of $T$ joint realizations for $B$ and $v$ represent the joint distribution of $B$ and $v$.

The following proposition extends Proposition 2 to this more general class of games. Additionally, it shows that a function does not affect the computational complexity if this function does not depend on $B$, nor on the coalition $S$. Again, we obtain the allocation of the Shapley value with a computational complexity that depends linearly on the number of players as well as the number of observations.

Proposition 3. In the setting described above, let each $\hat{v} \in \mathscr{V}$ be separable as

$$
\hat{v}(S)=E_{B \mid \hat{v}}\left[f_{\hat{v}}\left(B_{S}\right)\right] g_{\hat{v}}
$$

where the expectation is with respect to the realization of $B$ conditional on the event $\{v=\hat{v}\}, B_{S}$ is the restriction of $B$ to the players in $S \in 2^{N}, f_{\hat{v}}:[0,1]^{|S|} \rightarrow \mathbb{R}^{2}$ and $g_{\hat{v}}$ is a constant or a function that may depend on the realization $\hat{v}$ of $v$, but does not depend on $S$ or the realization of $B$. The Shapley value of $(N, \bar{v})$ is then

$$
\phi(\bar{v})=E_{v}\left[\phi\left(v^{f}\right) g_{v}\right]
$$

where for each $\hat{v} \in \mathscr{V}$

$$
\hat{v}^{f}(S)=E_{B \mid \hat{v}}\left[f_{\hat{v}}\left(B_{S}\right)\right]
$$

Roughly speaking, the Shapley value is simply a weighted average of Shapley values where the weight $P(v=\hat{v})$ is attached to the Shapley value of the game $(N, \hat{v})$. The factor $g_{\hat{v}}$ is only a scaling factor for the Shapley value of $(N, \hat{v})$.

Proof. The characteristic function of the composed game $(N, \bar{v})$ can be written as

$$
\bar{v}(S)=E_{v}[v(S)]=E_{v}\left[v^{f} g_{v}\right]
$$

\footnotetext{
${ }^{1}$ Here, we do not need to assume that the realizations of $B$ represent its probability distribution.

${ }^{2}$ This is a slight abuse of notation as the number of arguments of $f_{\hat{v}}$ depends on the number of players in $S$. Nevertheless, this should not introduce any confusion. Otherwise, one could define $f_{\hat{v}}$ on $[0,1]^{n}$ and impose that $f_{\hat{v}}$ does not depend on $B_{i}$ if $i \notin S$.
} 
where $\hat{v}^{f}(S)=E_{B \mid \hat{v}}\left[f_{\hat{v}}\left(B_{S}\right)\right]$. By the linearity of the Shapley value and the linearity of the expectations operator:

$$
\phi(\bar{v})=\phi\left(E_{v}\left[v^{f} g_{v}\right]\right)=E_{v}\left[\phi\left(v^{f}\right) g_{v}\right]
$$

This proposition is easily generalized to linear characteristic functions $\bar{v}(S)=\sum_{\hat{v} \in \mathscr{V}} \alpha_{\hat{v}} \hat{v}(S)$. The corresponding Shapley value equals $\phi(\bar{v})=\sum_{\hat{v} \in \mathscr{V}} \alpha_{\hat{v}} \phi\left(\hat{v}^{f}\right) g_{\hat{v}}$. Proposition 3 is then the special case $\alpha_{\hat{v}}=P(v=\hat{v})$.

It is known for each of the $T$ realizations of $B$ to which game $(N, \hat{v})$ it belongs. Therefore, the computational complexity of $\phi(\bar{v})$ increases linearly in $n$, the number of players, and in $T$, the number of realizations of $B$.

\section{Example}

Let the set $N_{\hat{v}}$ contain the non-null players in the game $(N, \hat{v})$. There are at least 2 players in this set for each $\hat{v} \in \mathscr{V}$. Only a null player does not affect $\kappa_{S}=\sum_{i \in S} B_{i}$ for any $S \in 2^{N}$. Thus $\kappa:=\kappa_{N}=\kappa_{\hat{N}_{\hat{v}}}$ and $P\left(B_{i}=0\right)=1$ if and only if $i \notin N_{\hat{v}}$. The joint distribution of $B$ and $v$ is known. This means that after selecting a characteristic function $\hat{v} \in \mathscr{V}$, the probability distribution of $B$ in the game $(N, \hat{v})$ is known. Let each $\hat{v} \in \mathscr{V} \mathrm{admit}^{3}$

$$
\hat{v}(S)=\frac{E_{B \mid \hat{v}}\left[\kappa_{S}\left(\kappa_{S}-1\right)\right]}{\left|N_{\hat{v}}\right|-1}
$$

Since $\bar{v}(S)=E_{v}[v(S)]$, the worth of the game $(N, \bar{v})$ equals

$$
\bar{v}(N)=E_{v, B}\left[\frac{\kappa(\kappa-1)}{\left|N_{v}\right|-1}\right]
$$

We define a dependence measure to measure player $i$ 's dependence on other players. This measure is the fraction of other participating non-null players given that player $i$ is a participating player: ${ }^{4}$

$$
\xi_{i}:=E_{v, B}\left[\frac{\kappa-1}{\left|N_{\hat{v}}\right|-1} \mid B_{i}=1\right]
$$

The vector $\xi$ contains this dependence measure for each player $i \in N$. We show that the Shapley value of player $i$ equals the product of the probability on the event $\left\{B_{i}=1\right\}$ and the dependence measure $\xi_{i}$ :

$$
\phi_{i}(\bar{v})=P\left(B_{i}=1\right) \xi_{i}=E_{v, B}\left[\frac{B_{i}(\kappa-1)}{\left|N_{v}\right|-1}\right]
$$

As a consequence, the dependence measure $\xi_{i}$ is related to the Shapley value in such a way that the computational complexity of both $\phi(\bar{v})$ and $\xi$ is linear in the number of players. We show this result by means of $(i)$ Proposition 2 and 3 as well as (ii) for a specific numerical example.

\footnotetext{
${ }^{3}$ Because this $\hat{v}$ is convex in $\kappa_{S}$, it follows that the game $(N, \hat{v})$ is convex such that the Shapley value is in the core (see Prop. 18.AA.1 in Mas-Colell et al [1995]). This means that no coalition $S \in 2^{N}$ can collectively increase their allocation by playing $(S, v)$.

${ }^{4}$ This measure is closely related to the systemic importance index in Zhou |2010|.
} 
(i) Notice that $\hat{v}(S)=w_{\hat{v}}(S)+z_{\hat{v}}(S)$ for the separable functions

$$
w_{\hat{v}}(S)=E_{B \mid \hat{v}}\left[\kappa_{S}^{2}\right] g_{\hat{v}} \quad z_{\hat{v}}(S)=-E_{B \mid \hat{v}}\left[\kappa_{S}\right] g_{\hat{v}}
$$

where $g_{\hat{v}}=\left(\left|N_{\hat{v}}\right|-1\right)^{-1}$. By Proposition 2, the Shapley value of player $i$ associated with the characteristic function

$$
w_{\hat{v}}^{f}(S)=E_{B \mid \hat{v}}\left[\kappa_{S}^{2}\right]
$$

is given by:

$$
\phi_{i}\left(w_{\hat{v}}^{f}\right)=E_{B \mid \hat{v}}\left[B_{i} \kappa\right]
$$

Using $\bar{w}(S)=E_{v}\left[w_{v}(S)\right]$, it follows from Proposition 3 that $g_{\hat{v}}$ can be interpreted as a scaling factor in determining the Shapley value of $\bar{w}$ :

$$
\phi_{i}(\bar{w})=E_{v}\left[\phi_{i}\left(w_{v}^{f}\right) g_{v}\right]=E_{v, B}\left[\frac{B_{i} \kappa}{\left|N_{v}\right|-1}\right]
$$

Similarly, for $z$ :

$$
\phi_{i}(\bar{z})=-E_{v, B}\left[\frac{B_{i}}{\left|N_{v}\right|-1}\right]
$$

The linearity of the Shapley value now gives the desired result (4).

(ii) We confirm the result in 4 for the following numerical example. In the game $(N, \bar{v})$ with $n=3$ players, the characteristic function is given by $(3)$. The game is over $T=50$ equally weighted periods. Player 3 is a null player during the first 20 periods, which means that the corresponding characteristic function $v_{1}$ is given by $(3)$ with $N_{v_{1}}=\{1,2\}$. In period 21, player 3 enters and remains in the game. The characteristic function $v_{2}$ is then given by 3 with $N_{v_{2}}=\{1,2,3\}$. Table 1 contains the realizations of $B$ and $v$ for this game. Intuitively,

\begin{tabular}{|c|c|c|c|c|c|c|c|}
\hline & $B_{1}$ & $B_{2}$ & $B_{3}$ & $\kappa$ & $v$ & $N_{v}$ & $\frac{\kappa-1}{\left|N_{v}\right|-1}$ \\
\hline$t_{1}$ & 1 & 1 & 0 & 2 & $v_{1}$ & $\{1,2\}$ & 1 \\
\hline$t_{2}$ & 0 & 1 & 0 & 1 & $v_{1}$ & $\{1,2\}$ & 0 \\
\hline other $t \in\{1, \ldots, 20\}$ & 0 & 0 & 0 & 0 & $v_{1}$ & $\{1,2\}$ & -1 \\
\hline$t_{3}$ & 1 & 0 & 0 & 1 & $v_{2}$ & $\{1,2,3\}$ & 0 \\
\hline$t_{4}$ & 1 & 1 & 1 & 3 & $v_{2}$ & $\{1,2,3\}$ & 1 \\
\hline$t_{5}$ & 1 & 0 & 1 & 2 & $v_{2}$ & $\{1,2,3\}$ & $1 / 2$ \\
\hline other $t \in\{21, \ldots, 50\}$ & 0 & 0 & 0 & 0 & $v_{2}$ & $\{1,2,3\}$ & $-1 / 2$ \\
\hline
\end{tabular}
player 3 contributes most to the total value since player 3 participates always simultaneously with another player. Table 2 provides for each $S \in 2^{N}$ the outcomes of the characteristic function $v_{1}(S)$ and $v_{2}(S)$.

Table 1: Realizations for the participation vector $B$ and the characteristic function $v$. The integer time periods are ordered by $1 \leq t_{1}<t_{2} \leq 20<t_{3}<t_{4}<t_{5} \leq 50$. 
Table 2: Outcomes for $\hat{v}(S)=\frac{E_{B \mid \hat{v}}\left[\kappa_{S}\left(\kappa_{S}-1\right)\right]}{\left|N_{\hat{v}}\right|-1}$ as in 3$\}$

\begin{tabular}{lcc}
\hline$S$ & $v_{1}$ & $v_{2}$ \\
\hline$\emptyset$ & 0 & 0 \\
$\{1\}$ & 0 & 0 \\
$\{2\}$ & 0 & 0 \\
$\{3\}$ & 0 & 0 \\
$\{1,2\}$ & $1 / 10$ & $1 / 30$ \\
$\{1,3\}$ & 0 & $2 / 30$ \\
$\{2,3\}$ & 0 & $1 / 30$ \\
$\{1,2,3\}$ & $1 / 10$ & $4 / 30$ \\
\hline
\end{tabular}

Using $(1)$, the Shapley value distributes $v_{1}(N)=\frac{1}{10}$ and $v_{2}(N)=\frac{4}{30}$ as follows among the players:

$$
\begin{aligned}
& \phi_{1}\left(v_{1}\right)=\frac{2 \cdot 0+1 \cdot(1 / 10-0)+1 \cdot(0-0)+2 \cdot(1 / 10-0)}{6}=\frac{1}{20} \\
& \phi_{2}\left(v_{1}\right)=\frac{2 \cdot 0+1 \cdot(1 / 10-0)+1 \cdot(0-0)+2 \cdot(1 / 10-0)}{6}=\frac{1}{20} \\
& \phi_{3}\left(v_{1}\right)=\frac{2 \cdot 0+1 \cdot(0-0)+1 \cdot(0-0)+2 \cdot(1 / 10-1 / 10)}{6}=0 \\
& \phi_{1}\left(v_{2}\right)=\frac{2 \cdot 0+1 \cdot(1 / 30-0)+1 \cdot(2 / 30-0)+2 \cdot(4 / 30-1 / 30)}{6}=\frac{1}{20} \\
& \phi_{2}\left(v_{2}\right)=\frac{2 \cdot 0+1 \cdot(1 / 30-0)+1 \cdot(1 / 30-0)+2 \cdot(4 / 30-2 / 30)}{6}=\frac{1}{30} \\
& \phi_{3}\left(v_{2}\right)=\frac{2 \cdot 0+1 \cdot(1 / 30-0)+1 \cdot(2 / 30-0)+2 \cdot(4 / 30-1 / 30)}{6}=\frac{1}{20}
\end{aligned}
$$

We use that $\phi_{i}(\bar{v})=E_{v}\left[\phi_{i}(v)\right]=\frac{20}{50} \phi_{i}\left(v_{1}\right)+\frac{30}{50} \phi_{i}\left(v_{2}\right)$ to obtain the second column in Table 3 . Hence, the computations require $O\left(n 2^{n} T\right)$ computations. The third and fourth column in Table 3 follow from Table 1 . but require only $O(n T)$ computations. Indeed, player 3 has the largest dependence measure $\xi_{i}$. It follows that the second column is equal to the product of the third column and the fourth column, as predicted by 44. This means that the Shapley value is obtained in $O(n T)$ computations.

Table 3: Shapley value, participation probabilities, and the dependence measure

\begin{tabular}{lccc}
\hline Player & $\phi_{i}(\bar{v})$ & $P\left(B_{i}=1\right)$ & $\xi_{i}$ \\
\hline 1 & $1 / 20$ & $4 / 50$ & $5 / 8$ \\
2 & $1 / 25$ & $3 / 50$ & $2 / 3$ \\
3 & $3 / 100$ & $2 / 50$ & $3 / 4$ \\
\hline
\end{tabular}

\section{References}

Algaba E, Bilbao JM, Fernández García JR, López JJ (2003) Computing power indices in weighted multiple majority games. Math Soc Sci 46:63-80

Castro J, Gómez D, Tejada J (2009) Polynomial calculation of the Shapley value based on sampling. Comput Oper Res 36:1726-1730 
Deng X, Papadimitriou CH (1994) On the complexity of cooperative solution concepts. Math Oper Res 19:257-266

Faigle U, Kern W (1992) The Shapley value for cooperative games under precedence constraints. Int J Game Theory 21:249-266

Fatima SS, Wooldridge M, Jennings NR (2008) A linear approximation method for the Shapley value. Artif Intell 172:1673-1699

Granot D, Kuipers J, Chopra S (2002) Cost allocation for a tree network with heterogeneous customers. Math Oper Res 27:647-661

Kalai E, Samet D (1987) On weighted shapley values. Int J Game Theory 16:205-222

Littlechild S, Owen G (1973) A simple expression for the Shapley value in a special case. Manag Sci 20:370-372

Mas-Colell A, Whinston MD, Green JR (1995) Microeconomic theory. Oxford University Press New York

Megiddo N (1978) Computational complexity of the game theory approach to cost allocation for a tree. Math Oper Res 3:189-196

Myerson RB (1980) Conference structures and fair allocation rules. Int J Game Theory 9:169-182

Shapley LS (1953) A value for $n$-person games. In: Kuhn H, Tucker A (eds) Contributions to the Theory of Games, vol 2, Princeton University Press, pp 307-317

Winter E (2002) The Shapley value. In: Aumann RJ, Hart S (eds) Handbook of Game Theory with Economic Applications, vol 3, Elsevier, chap 53, pp 2025-2054

Zhou C (2010) Are banks too big to fail? Measuring systemic importance of financial institutions. Int J Cent Bank 6:205-250 
Publisher:

CPB Netherlands Bureau for Economic Policy Analysis

P.O. Box $80510 \mid 2508$ GM The Hague

$\mathrm{T}(070) 3383380$

August 2011 | ISBN 978-90-5833-524-1 